\title{
THEORIE DE LA SEDUCTION GENERALISÉE ET TOPIQUE DU CORPS
}

Isabelle Gernet ${ }^{*}$

\begin{abstract}
RÉSUMÉ. Cet article propose une mise en perspective entre certaines propositions tirées de la théorie de la séduction généralisée de J. Laplanche et les théorisations en psychosomatique à propos du statut du corps. De cette analyse, il ressort que la référence au corps dans la genèse de la séduction pose la question d'une topique psychique organisée autour du clivage. L'hypothèse d'un clivage de la topique permet de rendre compte de la coexistence de secteurs hétérogènes du fonctionnement psychique. La discussion proposée examinera la pertinence de défendre une conception de la genèse de la pensée, à partir du modèle traductif, ancrée dans l'expérience du corps.
\end{abstract}

Mots clés: Clivage; corps; théorie de la séduction généralisée.

\section{TEORIA DA SEDUÇÃO GENERALIZADA E A TÓPICA DO CORPO}

RESUMO. O presente artigo procura pôr em perspectiva algumas propostas sobre o estatuto do corpo formuladas tanto pela teoria da sedução generalizada de Jean Laplanche como pelas teorias em psicossomática. Desta análise devemos ressalvar que a referência ao corpo na gênese da sedução levanta a questão de uma tópica psiquíca organizada em torno da clivagem. A hipótese de uma clivagem da tópica permite-nos explicar a coexistência de sectores heterogêneos do funcionamento psíquico. Na discussão aqui promovida examinaremos a pertinência da defesa de uma concepção da gênese do pensamento, a partir do modelo tradutivo, que se apoie na experiência do corpo.

Palavras-chave: Clivagem; corpo; teoria da sedução generalizada.

\section{THEORY OF GENERALIZED SEDUCTION AND THE BODY}

\begin{abstract}
This article presents a discussion between the theory of the generalized seduction of J. Laplanche and psychosomatics theorizations on the body. The analysis reveals that including the body in the genealogy of seduction questions the existence of psychic topics based on splitting. The hypothesis of a topic structured by the psychic mechanism of splitting underlines the coexistence of heterogeneous sectors of the mind. The discussion insists on the importance of defending a theory of the thought based on the translating model in its relations with the body.
\end{abstract}

Key words: Splitting; body; theory of the Generalized Seduction.

\section{TEORÍA DE LA SEDUCCIÓN GENERALIZADA Y LA TÓPICA DEL CUERPO}

RESUMEN. Este artículo busca poner en perspectiva algunas propuestas acerca del estatuto del cuerpo formuladas tanto por la teoría de la seducción generalizada de Jean Laplanche, como por las teorías en psicosomática. De este análisis debemos resaltar que la referencia al cuerpo, en la génesis de la seducción levanta la cuestión de una tópica psíquica organizada alrededor del clivaje. La hipótesis de un clivaje de la tópica nos permite explicar la coexistencia de sectores heterogéneos del funcionamiento psíquico. En esta discusión examinaremos la pertinencia de la defensa de una concepción del génesis del pensamiento, a partir del modelo traductivo, que se apoye en la experiencia del cuerpo.

Palabras-clave: Clivaje; cuerpo; teoría de la seducción generalizada.

Psychologue clinienne, Maître de Conférences en Psychologie, Université Paris Descartes. 
Le travail clinique auprès des malades somatiques pose des problèmes théoriques et pratiques à la psychanalyse, que le déploiement de la psychosomatique depuis les années 1960 à partir des travaux princeps de P. Marty et M. Fain dans le cadre de l'Ecole Psychosomatique de Paris (IPSO) a permis de révéler. Nous serons conduits, à partir d'un bref rappel des principales propositions théoriques en psychosomatique à interroger le statut du corps dans la clinique psychosomatique à la lumière de la théorie de la séduction généralisée. Ce qui se présente comme une clinique du défaut de mentalisation et de la répression des affects bénéficie en effet de la référence à la séduction originaire pour rendre compte de la genèse du processus d'affectation, qui n'est pas endogène, mais initié par l'implantation du sexuel adulte dans le corps de l'enfant.

\section{LE STATUT DU CORPS EN PSYCHOSOMATIQUE}

Depuis les premières descriptions concernant les rapports entre l'apparition de maladies somatiques et des discontinuités du fonctionnement psychique, par les psychosomaticiens de l'Ecole de Paris, il est fréquent d'identifier comme caractéristique la sémiologie mentale négative qui accompagne les atteintes somatiques. En effet, chez les sujets présentant des atteintes somatiques, les formations psychiques comme les fantasmes, les rêves, la pensée associative, seraient absentes ou fortement altérées. L'accrochage au factuel, au perceptif seraient les témoins de la recherche massive d'étayage sur les objets et la réalité, mais seraient également, pour certains auteurs, la résultante de défenses massives établies par ces sujets pour lutter contre la menace de désorganisation de l'appareil psychique par l'angoisse. Chez les malades somatiques, les possibilités de transformation des excitations venant du corps biologique, au profit de l'expressivité pulsionnelle érotique et agressive feraient défaut, ce qui conduirait à désigner ces manifestations comme caractéristiques d'une clinique de la désaffectation pour reprendre ici l'expression de Joyce McDougall (1989). L'absence de manifestation affective viendrait également infiltrer l'établissement de la névrose de transfert ce qui a conduit Marty et M'Uzan (1963) à qualifier la relation thérapeutique qui s'instaure avec le malade de relation blanche. Les ruptures et atteintes du processus de mentalisation et donc de symbolisation de l'angoisse de la part des malades somatiques s'actualiseraient dans les éprouvés contre-transférentiels de l'ordre de la neutralisation, voire de la minéralisation du fonctionnement psychique du clinicien (Dejours,
2009). Les affects seraient non seulement absents du discours du sujet, mais seraient même empêchés et étouffés chez le clinicien. Dans certains cas, l'apparition de sentiment de vidages ou parfois de somatisations chez l'analyste signeraient le mouvement de clivages temporaires, résultant de la relation tout-à-fait spécifique établie avec le patient (Bayle, 1988). Cette neutralisation du fonctionnement psychique de l'autre aurait à voir avec la violence de vécus du corps n'ayant pas été pris en charge par le psychisme du patient et permettant de leur accorder un statut d'éprouvés. Le recours massif à la répression, qui consiste à empêcher le surgissement des pensées, serait pour la plupart des auteurs la conséquence de l'état de privation ou de déficit psychique qui serait caractéristique des malades somatiques. La répression, qui a pu être envisagée comme le refoulement du pauvre (Press, 1995) révèlerait les altérations de l'appareil psychique tant au niveau du masochisme érogène que du narcissisme. L'utilisation prématurée et élargie de la répression serait en effet comprise comme une tentative de faire face au défaut de pareexcitation. En court-circuitant le refoulement, la répression contribuerait à l'altération, voire à la disparition de l'activité fantasmatique exposant alors le corps biologique au risque des tendances destructrices.

Les descriptions sur les avatars de l'affect en psychosomatique ont contribué à réexaminer à nouveaux frais le rôle de processus psychiques comme le clivage qui aboutissent à la coexistence de secteurs hétérogènes dans le fonctionnement psychique. Il apparaît en effet que les sujets disposent de solutions pour s'empêcher de ressentir l'angoisse et de souffrir, solutions qui ont pour effet de couper le moi d'une partie de la réalité et ont pour corollaire une amputation, voire une fragmentation du moi. "Le moi est donc clivable" écrit Freud (1932/1995, p. 141). "Il se clive au cours d'un bon nombre de ses fonctions, au moins provisoirement. Les fragments peuvent se réunir de nouveau par la suite" (Freud, 1932/1995, p.141). Mais, en 1938 il reprend:

\footnotetext{
C'est donc un conflit entre la revendication de la pulsion et l'objection opposée par la réalité. Or l'enfant ne fait ni l'un ni l'autre, ou bien il fait simultanément l'un et l'autre, ce qui revient au même. Il répond au conflit par deux réactions opposées, toutes deux valables et efficaces (...) Les deux parties en lutte y ont trouvé leur compte (...) Ce succès fut atteint au prix d'une déchirure dans le moi, déchirure qui ne guérira plus jamais, mais s'agrandira avec le temps (Freud, 1938/2010, p. 222).
} 
Pour Freud (1938/2010), le clivage du moi consiste donc avant tout en une réponse élaborée pour conserver le rapport à la réalité, qui conditionne le maintien de la représentation de l'objet et du lien à celui-ci. Le clivage, en occasionnant un traitement spécifique de la réalité, constitue également une manière de contredire la perception.

A partir de la clinique psychosomatique on est toutefois conduit à situer le problème en des termes sensiblement différents: avant que de s'interroger sur les conditions de la perte de la réalité, l'expérience de la maladie somatique pose le problème de la perte du rapport à soi et des éprouvés du moi. En d'autres termes, il s'agit de comprendre ce qui entrave la capacité du sujet à être affecté par son corps et par l'autre. Ce défaut de la disposition sensible du corps contraste avec la symptomatologie somatique parfois bruyante qui peut venir engager, dans certains cas, le pronostic vital. Dans le travail clinique avec ces patients, une place centrale revient au traitement psychique $^{1}$ des états du corps qui renseigne sur la manière dont ces manifestations du corps peuvent constituer, ou non, une exigence de travail pour la pensée. Pour la plupart des cliniciens prenant en charge des malades somatiques, la visée du travail thérapeutique est donc orientée vers l'exigence de représenter ce qui vient du corps. La représentation aurait elle-même ses exigences et contraintes, que la clinique psychosomatique permettrait de révéler, à travers l'impossibilité de la part du psychisme à métaboliser, décoder, ou encore à traduire les états du corps (Aisenstein, 2010). Toute la difficulté du travail clinique avec ces patients immobilisés dans le registre de l'actuel serait de favoriser les conditions pour que le corps et son expérience puissent (re)devenir un objet d'interprétation dans le cadre du travail thérapeutique. C'est d'ailleurs cette particularité clinique qui a conduit les auteurs de l'IPSO à privilégier la perspective économique dans l'abord des patients somatiques, et qui a été récapitulé à travers la formule devenue célèbre de la bêtise du symptôme somatique (M’Uzan, 1997). L'impossibilité des malades somatiques à rêver serait là encore un témoin de l'échec du travail de représentation et de traitement

1 En référence au texte de Freud (1890/1984), intitulé Le traitement psychique (traitement d'âme), qui envisage la question des fondements corporels des processus de pensée: "Les affects au sens restreint se caractérisent par un rapport tout-à-fait particulier aux processus corporels; mais en toute rigueur, tous les états psychiques, y compris ceux que nous avons l'habitude de considérer comme des processus de pensée sont dans une certaine mesure affectifs et on n'en trouve aucun qui ne soit accompagné de manifestations corporelles et qui n'ait la faculté de modifier les processus corporels" (p. 7). des perceptions grâce à leur mise en latence par le préconscient.

Les théorisations en psychosomatique défendent donc, pour la plupart, la thèse de la psychisation du corps et de ses manifestations, en concevant les formations psychiques comme des productions qualitatives issues de la transformation de processus corporels caractérisés par leur dimension quantitative (Rosé, 1997; Smadja, 2011). Les malades somatiques ne pourraient pas bénéficier, au même titre que les sujets présentant une organisation psychonévrotique, de la qualité et de la souplesse des formations psychiques comme les fantasmes, les rêves ou les processus de pensée. Pourtant, ces conceptions ne permettent pas d'expliquer que des sujets qui présentent une organisation névrotique de la personnalité puissent être victimes d'une décompensation somatique, alors qu'ils ne présentent pas les défaillances psychiques habituelles des malades somatiques. Ces propositions peinent également à rendre compte des remaniements psychiques liés à une décompensation somatique en référence au point de vue dynamique qui conduit à accorder une place particulière à l'évolution du symptôme somatique plutôt qu'à sa genèse.

Les recherches en psychosomatique développées par C. Dejours ont privilégié cette voie et proposent un modèle topique articulé autour de la référence au clivage (troisième topique ou topique du clivage). Ce clivage, qui se prolonge jusque dans l'inconscient, rend possible le maintien de la santé physique et mentale et accorde à la théorie de la séduction généralisée de Laplanche une place capitale dans la constitution de la topique adossée à la genèse du corps érotique. La référence au corps érotique, qui désigne le corps éprouvé et engagé dans la relation avec l'autre, formé par subversion du biologique au profit de l'érotique (Dejours, 2001), permet notamment de rendre compte de la place singulière et de l'engagement $\mathrm{du}$ corps dans la dynamique transférentielle.

\section{THÉORIE DE LA SÉDUCTION GÉNÉRALISÉE ET GENÈSE DU CORPS ÉROTIQUE}

La genèse du corps érotique réside dans une remise au travail de la notion d'étayage, qui revêt une acception tout-à-fait singulière dans le cadre de la théorie de la séduction généralisée. La séduction est, pour J. Laplanche (1988), au principe de la naissance de la sexualité, ce qui lui confère son caractère indomptable et anarchique. C'est en raison de la situation anthropologique fondamentale, qui se caractérise par une inégalité foncière entre l'enfant et 
l'adulte, que les soins prodigués à l'enfant sont toujours contaminés par les fantasmes et l'inconscient de l'adulte. En d'autres termes, la communication vectorisée par l'attachement qui s'instaure entre l'adulte et l'enfant, constitue l'onde porteuse de messages compromis par le sexuel inconscient de l'adulte. Ces messages, infiltrés par le sexuel, qui ont un pouvoir excitant et donc de séduction, suscitent une exigence de traduction pour l'enfant, car celui-ci se retrouve face à des excitations qu'il ne peut maîtriser. Ces messages sont provocateurs chez l'enfant d'un mouvement de traduction. Mais cette tentative de symbolisation initiée par l'enfant comporte, comme toute traduction, des résidus qui seront à l'origine de la constitution du noyau de l'inconscient de l'enfant. Les efforts de traduction de l'enfant en vue de maîtriser l'excitation véhiculée par les messages restent en effet toujours incomplets et imparfaits. La constitution de l'inconscient à partir de ce qui n'a pas pu être métabolisé du message de l'autre se fait connaître sous la forme d'une "quintessence d'altérité" (Laplanche, 1999a, p. 137). La théorie traductive de l'inconscient proposée par J. Laplanche cherche par conséquent à rendre compte du caractère d'étrangèreté de l'inconscient et de la nature conflictuelle de ses émergences, qui prennent la forme des retours du refoulé.

La théorie traductive de l'inconscient sexuel refoulé, à partir de la généralisation de la séduction, se présente comme une forme de réponse au texte de S. Ferenczi (1932/1982) sur la "Confusion des langues entre l'adulte et l'enfant". Notons que pour Ferenczi, la séduction qui organise les rapports entre les adultes et l'enfant produirait un clivage dans l'organisation psychique de l'enfant, du fait de la massivité des excitations pulsionnelles auxquelles il serait confronté de manière trop précoce. Le traumatisme résulterait des mouvements passionnels des adultes envers l'enfant qui désavouent en même temps sa souffrance psychique. L'enfant, débordé, ne pourrait qu'observer l'événement traumatique à défaut de pouvoir le traiter psychiquement. Dans certains cas, cette situation conduirait au développement d'une hypersensibilité aux adultes, l'enfant pouvant se faire le psychiatre de ses parents ou devenir un nourrisson savant (Ferenczi, 1923/1974). En réponse à la menace de débordement traumatique se développerait une forme de pensée prématurée, une intelligence pure que Ferenczi conçoit comme le produit du "processus de l'imminence de la mort ou du moins de l'insensibilité psychique" (Ferenczi, 1932/1982, p. 287). Ces descriptions de Ferenczi insistent sur la valeur traumatique de la séduction mais laissent dans l'ombre ce qui, dans la situation de confusion, est susceptible de susciter une exigence de travail psychique.
Dans le cadre de la théorie traductive de l'inconscient, J. Laplanche (1988). insiste au contraire sur la relation fondamentalement asymétrique qui existe entre les adultes et l'enfant, mais choisit de privilégier, à la place de la confusion mise en avant par Ferenczi, la traduction des messages adressés à l'enfant par les adultes. La sexualité est de ce fait implantée dans le corps de l'enfant et procède d'une origine extérieure, par l'autre, c'est-à-dire par les adultes.

Le déséquilibre entre l'adulte et l'enfant, généré par la séduction qui conduit à l'apparition du sexuel infantile, est au principe de l'étayage de la pulsion sur la fonction physiologique. Ce faisant, l'étayage n'apparaît plus comme un processus endogène mais trouve sa vérité au regard de la situation de la séduction. Grâce à l'étayage, la pulsion se dégage au moins partiellement de l'instinct et aboutit à l'installation du primat du désir sur le registre des besoins. L'étayage de la pulsion sur les fonctions physiologiques (organes des sens, sphincters, muqueuses, organes moteurs, etc.) fonctionnerait comme une subversion de l'ordre physiologique au profit de l'économie érotique aboutissant à la genèse du corps érotique. Le corps érotique qui désigne le corps éprouvé et engagé dans la relation avec l'autre ne serait donc pas réductible au corps biologique des régulations physiologiques. A ce titre, la clinique des malades somatiques renseigne de manière originale sur les altérations du corps érotique, les ruptures, ou les interruptions de la subversion libidinale.

\section{ECHECS DE LA TRADUCTION OU ACCIDENTS DE LA SÉDUCTION?}

La construction du corps érotique par la subversion du corps biologique s'opère à travers le corps à corps entre l'adulte et l'enfant, mais est aussi marquée par les impasses de la relation. Alors que la traduction opérée par l'enfant des messages comporte toujours une part résiduelle, qui est au principe de l'inconscient sexuel refoulé, il arrive que certains messages des adultes soient totalement intraduisibles de la part de l'enfant, ce que C. Dejours (2004) désigne comme des accidents de la séduction. Les soins du corps de l'enfant par l'adulte éveillent chez lui des réactions variées. La manipulation du corps de l'enfant suscite en effet chez l'adulte des fantasmes et des affects, jusqu'à pouvoir parfois déclencher de l'angoisse. Dans certains cas, l'adulte ne pourrait supporter l'excitation née du corps à corps avec l'enfant, il chercherait alors à la maîtriser, voire à la stopper de façon plus ou moins brutale. Ces réactions de gêne, de dégoût, et parfois de haine vis-à-vis du 
corps de l'enfant, manifestées par l'adulte, auraient pour effet de déborder massivement les capacités de traduction de l'enfant. Ce dernier se trouverait dans l'impossibilité de penser ce qui se produit dans son corps (Debray, Dejours \& Fedida, 2002), entraînant une interruption du processus de subversion libidinale. Certaines zones, électivement sollicitées dans la relation entre l'adulte et l'enfant seraient dépourvues de leur potentialité érogène, et ne pourraient donner lieu à aucun travail de pensée lorsqu'elles se trouveraient à nouveau convoquées dans les échanges intersubjectifs ultérieurs. Le processus de traduction est ici entravé, du fait du comportement de l'adulte qui interrompt la communication, et ne donnerait donc lieu à aucune formation de refoulé. Les zones exclues de la subversion libidinale sont à l'origine d'amputations du corps érotique qui se traduiraient par une agénésie pulsionnelle. Ces zones froides, dénuées de potentialité érogène, car exclues de la subversion libidinale, seraient amenées à se cristalliser au cours du développement sous une forme différente de celle qui organise le processus du refoulement et l'inscription des expériences dans l'inconscient. Ces expériences non relayées par la symbolisation sont proscrites conduisant à la formation d'un inconscient dont la nature différerait de l'inconscient sexuel refoulé. Cet inconscient sans pensée, C. Dejours (2001) l'appelle inconscient amential, en référence aux descriptions de Meynert sur la confusion mentale qui s'accompagne de la suspension de toute pensée, et J. Laplanche proposera de le désigner plutôt comme un inconscient enclavé (Laplanche, 2006). Il apparaît que la différence de terminologie désignant ce secteur de l'inconscient réside principalement dans une divergence accordée au destin des messages énigmatiques.

Dans le cadre de la théorie de la séduction, Laplanche (1993/1999b) insiste sur l'effet d'instabilité et de déliaison généré par l'implantation du message, où la traduction avec refoulement est toujours un remaniement du message: tout message est "partiellement traduit et partiellement refoulé" (p. 82). Il ne pourrait exister de ce fait de message non traduit résultant d'impasses de la séduction, mais tout message non traduit resterai en attente d'une nouvelle traduction. Laplanche distingue deux types de processus qui organisent la transmission des messages: l'implantation et sa variante violente qui est l'intromission. Si le processus d'implantation est au principe de la séduction et au fondement de l'organisation psychique normalo-névrotique, Laplanche attribue à l'intromission la constitution de messages de type persécutif qui signent l'échec de la dynamique "traductive-refoulante" (Laplanche, 1990/1997b, p. 358). Cette variante violente de l'implantation qu'est l'intromission ne sera évoquée que de manière programmatique par Laplanche, mais sera reprise essentiellement par deux auteurs s'appuyant sur la théorie de la séduction généralisée: L. C. Tarelho (1999) dans une relecture de la paranóia et M. R. Cardoso (1993) dans une thèse originale sur le Surmoi. Ces deux auteurs privilégient la voie de la violence de la séduction pour rendre compte de la formation d'enclaves psychotiques, distinctes des résidus de la traduction. Ces enclaves non métabolisées se caractériseraient par une résistance particulière au processus du refoulement qui institue le mouvement de structuration du psychisme, s'avérant en quelque sorte rebelles à toute forme de traduction. L'intraduisible du message se ferait connaître par une circulation menaçante pour la topique psychique des éléments à la dérive se manifestant, dans le cas de la psychose paranoïaque par un retour du message intraduisible sous la forme hallucinatoire, et sous la forme des impératifs tyranniques et arbitraires dans le cas du Surmoi. Bien que Tarelho comme Rezende Cardoso relèvent que les messages, même sous leur forme violente, transitent par le corps, ils privilégient tous deux la référence à la défaillance des codes de traduction transmis par les adultes à l'enfant.

Pour Laplanche (2007) en effet, le caractère énigmatique des messages compromis par le sexuel inconscient de l'adulte ne réside pas dans le fait que l'enfant ne possède pas le code, mais qu'il ne dispose pas des moyens adéquats pour constituer un code substitutif. Le code, dont le complexe de castration et le complexe d'Edipe en sont les formes les plus communes, est proposé par l'environnement social à l'enfant et remplit le rôle d'une aide à la traduction des messages énigmatiques (Laplanche, 2007). Cette question du code sera travaillée, à la suite des propositions de Laplanche, d'une manière originale par Bleichmar (2000). Selon elle, les adultes n'adressent pas seulement un message à l'enfant, mais lui transmettent également des éléments de liaison qui seraient indispensables pour que l'enfant puisse être en mesure de métaboliser le caractère énigmatique du message adulte. Bleichmar est ainsi conduite à insister sur la fonction narcissisante de la mère en rapatriant, dans la conception de la genèse de l'inconscient à l'aune de la théorie de la séduction généralisée, les travaux psychanalytiques portant notamment sur les enveloppes psychiques. L'écueil principal du modèle de Bleichmar, adossé sur la genèse des limites de l'appareil psychique, est qu'il privilégie le mouvement de psychisation à partir de l'expérience corporelle au détriment d'une théorie traductive de l'inconscient et de la pensée, en tant que formations initiées par la séduction originaire. 
Les travaux qui cherchent à rendre compte des destins de la traduction dans la voie ouverte par la théorie de la séduction généralisée de Laplanche achoppent donc sur l'intégration du corps dans la genèse de la topique psychique à partir d'une forme d'édulcoration des effets de la violence et des impasses de la séduction.

Une définition restrictive de la violence - ce qui permet notamment de la distinguer de l'agressivité - permet de la caractériser d'abord par des conduites (du sujet lui-même ou des autres) qui usent de contraintes par la force et visent spécifiquement le corps. L'implication du corps dans les conduites violentes est de ce fait primordiale et permet de comprendre les répercussions psychiques de la violence parfois redoutables sur la subjectivité. La place à accorder à la violence dans le modèle traductif de Laplanche se présente dès lors comme un chaînon intermédiaire majeur en vue de dégager les conditions propices au déploiement d'une topique du corps. L'insistance de Laplanche (2004, 2007) sur l'altérité radicale constitutive de la subjectivité, faisant du sujet l'objet d'un autre en raison de la séduction, le conduira toutefois à évoquer à plusieurs reprises la violence (notamment dans le cas de la séduction violente agie par l'adulte) et se trouvera finalement problématisé en termes d'échec radical de la traduction, à partir d'une reprise du modèle de la troisième topique de C. Dejours.

Si Laplanche indique par ailleurs que la nature des messages n'est pas que verbale, que l'implantation des messages énigmatiques se fait dans le corps de l'enfant du fait de la polarisation de l'attention des adultes par la maturation physiologique de l'enfant, il reste toutefois réservé quant à la place à accorder au corps dans la dynamique de la séduction. Bien qu'il reconnaisse au travail de la cure, mais aussi à certaines constellations culturelles le pouvoir de remettre en cause le renfermement ptoléméique de l'appareil psychique, l'expérience du corps et de la maladie n'est pas indiquée comme une voie propice à la reprise de la dynamique de la symbolisation, à partir de la réouverture de la blessure de l'énigme générée par la séduction originaire. L'écueil principal de la référence au corps résiderait en effet dans le problème du biologisme dont est empreinte toute expérience du corps, avec le risque de perdre le caractère foncièrement disruptif du sexuel en le rabattant sur le registre de l'autoconservation (Laplanche, 1991/2006). Cette position radicale de Laplanche quant à l'essence du sexuel permet de se défaire du primat de la représentation en déconstruisant les modèles théoriques qui interprètent la représentation comme une trace de la perception, en référence au modèle de la réalisation hallucinatoire du désir indiquée par Freud. La radicalité de la théorie de la séduction conduit à défendre que l'instauration de l'inconscient produise une perte de toute référence et que les traces mnésiques soient comprises comme des déchets des processus de mémorisation signifiantsdésignifiés. Pour Laplanche (1993/1999b), la représentation "en devenant inconsciente, perd son statut de représentation (de signifiant) pour devenir une chose qui ne représente (ne signifie) plus qu'elle-même" (p. 76).

Dans ce cadre, l'attaque interne par la pulsion donne de l'angoisse et du corps une acception particulière:

L'angoisse (...) ce serait alors le moi livré à
la pulsion, débordé par elle, comme le
nourrisson -qui n'a pas encore de moi mais
qui est le moi - est livré au débordement
d'énergie interne. L'angoisse c'est donc le
moi livré à l'attaque interne ou, pour mieux
dire, à l'attaque interne-externe; quelque
chose qu'on peut imaginer comme implanté
dans son écorce, qu'il ne peut fuir et qu'il
doit tant bien que mal tenter d'assumer et qui
est la pulsion (Laplanche, 1980, p. 151-152).

L'implantation du sexuel requiert donc une sensibilité spécifique du corps qui éprouve. Laplanche propose dès lors la définition suivante de la pulsion et de l'affect:

La pulsion est la façon dont l'objet-source
affecte le corps et exige de lui un certain
travail. Que l'affect (et l'affect de base,
l'angoisse) soit du 'corps' ne me parait guère
contestable, ni même, nécessairement,
antifreudien. Reste que c'est seulement à
partir de la passivité du corps par rapport au
'corps étranger interne' que peut être
examiné dans quelle mesure l'affect doit être
dit effet, représentance, transposition ou
traduction de la pulsion ( Laplanche,
1989/1997a, p. 342).

La thèse de la théorie de la séduction généralisée nous conduit ainsi à réexaminer la conception des modalités singulières du fonctionnement psychique des malades somatiques abordées à partir du défaut ou des altération de l'activité de représentation et de mentalisation. En effet, la clinique psychosomatique nous conduit plutôt à apprécier ce qui, à partir de l'expérience du corps mobilisée par la rencontre intersubjective dans le cadre de la situation thérapeutique échoue à susciter une exigence de 
travail pour le moi et vient entraver le processus d'affectation (M'Uzan, 1970/1997).

\section{CORPS, TRADUCTION ET TRAVAIL DE PENSÉE}

Nous avons vu que la voie indiquée par Laplanche insiste sur la passivité du corps, en lien avec la position originaire du masochisme dans la vie psychique (Laplanche, 1991/1997c). La pulsion est indissociablement liée à la fantaisie inconsciente conçue comme résidu du message énigmatique, ce que Laplanche désigne comme le temps réfléchi (temps auto). La séduction, en tant que temps originaire constitutif de la topique psychique, détermine la passivité première due à l'effraction suscitée par la compromission du message qui déborde le registre de l'auto-conservation. Tout travail psychique résulte ainsi d'une opération consécutive à cet état de passivité originaire que désignent des verbes actifs spécifiques des processus psychiques comme "je projette, je dénie, je refoule, je forclos etc (..." (Laplanche, 1990/1997b, p. 357). Le travail de la pensée relève de l'unification narcissique réalisée par le moi dans sa tentative de trouver une nouvelle traduction au corps étranger interne qu'est la pulsion. Le devenir d'une grande partie de l'excitation sexuelle traumatisante est d'être liée, par la voie de la symbolisation, par le travail du moi investi de libido narcissique. La fantaisie inconsciente oriente les modes d'investissement de la pensée, en vue de trouver des formes figurées aux affects étrangers qui s'imposent au moi et menacent son intégrité. Le statut de la fantaisie dans la théorie de la séduction généralisée se présente cependant comme ambigu. En tant qu'objet-source de la pulsion, elle constitue une source d'excitation, trace de la séduction originaire, mais elle représente en même temps une tentative de liaison indissociablement liée à la pulsion. La fantaisie revêt donc aussi une fonction conservatrice pour la topique psychique, ce qui a été souligné notamment par Abraham et Torok (1987) qui envisagent le fantasme comme "toute représentation, toute croyance, tout état du corps (...) tendant au maintien du statu quo topique" (p. 260).

La clinique psychosomatique pose de manière centrale le problème de l'extinction de l'activité fantasmatique qui se manifeste par les vulnérabilités du corps érotique. Ces dernières seraient les héritières de la violence et de la haine de l'adulte contre le corps de l'enfant qui ont court-circuité les tentatives de traduction de l'enfant aboutissant à un clivage de la topique psychique. L'échec à traduire résiderait donc moins dans les caractéristiques ou contenus du message que dans la manière dont il est adressé à l'enfant. Parce que le corps est au principe du fantasme (objet-source de la pulsion), la violence, en prenant pour cible le corps, abolirait tout relais corporel pour l'excitation pulsionnelle. Les rejetons de la violence contre le corps de l'enfant se feraient connaître chez les malades somatiques par des états de vide affectif et des épisodes aux cours desquels ils disent ne plus rien sentir.

La déstabilisation du clivage se révèle toujours par une expérience du corps consécutive à la mobilisation intersubjective, dont la forme cardinale est, si l'on suit C. Dejours, la rencontre érotique. C'est d'abord la réalité de la rencontre avec l'autre qui est susceptible de déstabiliser le clivage organisant la topique psychique. Au cours des échanges amoureux, la mobilisation de zones dépourvues de sensibilité érogène va se traduire par l'extinction de l'activité fantasmatique et du désir. Ces zones du corps ne pourraient être à l'origine d'aucun développement de fantasmes conduisant à orienter et guider l'excitation. La sollicitation de ces zones pourrait au contraire conduire à déclencher une crise d'identité chez le sujet se traduisant par une décompensation psychopathologique. Dans certains cas, la rencontre avec l'autre induirait une menace de désubjectivation, où le sujet est confronté à l'expérience du vide, de l'absence de sensibilité de son corps, de la vie qui s'absente en soi.

Il s'avère en fin de compte que le clivage de l'inconscient qui résulte de cette topique du corps est silencieux, et ne devient visible que lorsqu'il est déstabilisé. En effet, l'inconscient amential, au contraire de l'inconscient sexuel refoulé ne se fait pas connaître par des formations de compromis, mais par des phénomènes pathologiques qui se distinguent des retours du refoulé : passage à l'acte (auto-hétéroagressif), poussée évolutive d'une maladie somatique, confusion mentale en seraient les formes les plus typiques. Mais comment le clivage peut-il rester silencieux en empêchant les émergences de l'inconscient amential qui viendraient menacer la stabilité de la topique ? Pour Dejours, cet inconscient est protégé par le système conscient, dont la sollicitation permet aux sujets d'éviter les décompensations par le recours privilégié et efficace à la réalité. Cela se traduit par un investissement important de la pensée logique et rationnelle qui est déconnectée de l'inconscient sexuel, proche de l'intelligence pure évoquée par Ferenczi, que C. Dejours désigne comme une pensée d'emprunt. La pensée d'emprunt, adossée à l'imaginaire social, correspond à la pensée opératoire décrite en psychosomatique par P. Marty et M. de M'Uzan (1963) et remplit chez les malades somatiques, une fonction défensive: celle de conjurer la menace de 
désorganisation du moi. En maintenant une adaptation à la réalité et un conformisme aux normes de comportements sociaux et professionnels, la pensée d'emprunt permet notamment de stabiliser la coexistence d'attitudes ou de pensées contradictoires, et ainsi de conjurer l'angoisse.

Si la fonction protectrice du clivage remplie par la pensée d'emprunt ne fait aucun doute, des questions se posent quant à sa genèse. A partir de quels processus psychiques, cette pensée d'emprunt désincarnée estelle élaborée et entretenue? C'est le plus souvent la référence au mécanisme psychique du contreinvestissement qui est convoqué pour rendre compte de la mise en place de processus psychiques défensifs, visant à lutter contre la menace de débordement pulsionnel et se traduisant par une forme de prématurité du moi ou de faux self. Dans le champ de la psychosomatique, on doit notamment à M. Fain d'avoir dégagé des hypothèses métapsychologiques sur la genèse du fonctionnement opératoire conçue comme la conséquence de la mise en place de modes de défenses précoces visant à protéger la topique psychique de la menace traumatique. L'élaboration prématurée d'un pare-excitation autonome, consécutif à l'action de la pulsion de mort, privilégiant le recours à la motricité et aux procédés auto-calmants, constituerait pour lui une impasse par rapport au travail de symbolisation (Fain, 1971). Ces remarques ouvrent sur des développements concernant le statut métapsychologique de la pulsion de mort non sexuelle résultant de la destruction de la perception sensible du corps par la violence. Nous avons vu que des pistes sont suggérées par les travaux de Ferenczi sur le traumatisme et l'élaboration de modes de défense précoces aboutissant à un clivage dans l'organisation psychique de l'enfant, pistes vis-à-vis desquelles la théorie de la séduction généralisée apporte un nouvel éclairage.

\section{CONCLUSION}

Les apports féconds de la théorie de la séduction généralisée contribuent à renouveler les conceptions psychanalytiques et psychosomatiques sur la pensée en suivant les destins du sexuel, mais en rendant compte également des vicissitudes du non-sexuel résultant des accidents de la séduction. La référence à une topique du corps qui s'en dégage ouvre des perspectives originales dans l'analyse de la genèse de conduites spécifiques qui s'originent dans l'insensibilité du corps résultant des accidents de la séduction. Parce que le clivage trouve sa genèse dans les amputations du corps érotique, il produit des défenses qui se caractérisent par une insensibilité à sa propre souffrance (mais également à celle d'autrui). Ce dernier point a des incidences pratiques dans la conduite du travail thérapeutique qui ne seront pas abordées ici faute de place. Il semble en effet que la conquête de la sensibilité du corps reste pour une part tributaire des risques encourus à affronter le clivage et les zones de vulnérabilité du corps érotique issues des accidents de la séduction, ce qui conduit à réexaminer, à la lumière du clivage de l'inconscient, le débat ouvert par Freud, dès 1905, sur les voies d'influence réciproques entre sexuel et non sexuel.

\section{REFERÊNCIAS}

Abraham, N., \& Torok, M. (1987). L'écorce et le noyau. Paris: Flammarion.

Aisenstein, M. (2010). Les exigences de la représentation. Revue Française de Psychanalyse, 74:1367-1392.

Bayle, G. (1988). Traumatismes et clivages fonctionnels. Revue Française de Psychanalyse, 52:1339-1356.

Bleichmar, S. (2000). La fondation de l'inconscient et la clinique de l'enfant. Paris: PUF.

Cardoso, M. (1993). Surmoi et théorie de la séduction généralisée. L'inquiétante étrangeté du Surmoi. Psychanalyse à l'Université. 18(71): 87-110.

Debray, R., Dejours, C., \& Fédida, P. (2002). Psychopathologie de l'expérience du corps. Paris: Dunod.

Dejours, C. (2001). Le corps d'abord. Paris: Payot.

Dejours, C. (2004). Le corps entre séduction et clivage. In J. Aïn. Résonances, entre corps et psyché. (pp. 59-83). Paris: Erès.

Dejours, C. (2009). Les dissidences du corps. Paris: Payot.

Fain, M. (1971). Prélude à la vie fantasmatique. Revue Française de Psychanalyse, 35(2-3): 291-364.

Ferenczi, S. (1974). Le rêve du nourrisson savant. In S. Ferenczi. Psychanalyse III. Euvres complètes, 19191926 (p. 203). Paris: Payot. (Original publié en 1923).

Ferenczi, S. (1982). Confusion de langue entre les adultes et l'enfant (pp.125-138). In Psychanalyse IV. Cuvres complètes, 1927-1933. Paris: Payot. (Original publié en 1932).

Freud, S. (1984). Traitement psychique (traitement d'âme) (pp. 1-23). In Freud, S. Résultats, idées, problèmes I, Paris: PUF. (Original publié en 1890).

Freud, S. (1995). La décomposition de la personnalité psychique. Nouvelles suites des leçons d'introduction à la psychanalyse (pp. 140-163). In Freud, S. CEuvres Complètes de Freud, Vol. XIX, Paris: PUF. (Original publié en 1932).

Freud, S. (2010). Le clivage du moi dans le processus de défense (pp.219-224). In Freud, S. Euvres Complètes de Freud, Vol. XX, Paris: PUF. (Original publié en 1938).

Laplanche, J. (1980). Problématiques I: L'angoisse. Paris: PUF. 
Laplanche, J. (1988). Nouveaux fondements pour la psychanalyse. Paris: PUF.

Laplanche, J. (1997a). Débat à propos de temporalité et traduction (pp. 337-353). In J. Laplanche. Le primat de l'autre en psychanalyse - Travaux 1967-1992. Paris: Flammarion. (Original publié en 1989).

Laplanche, J. (1997b). Implantation, intromission (pp. 355-358). In J. Laplanche. Le primat de l'autre en psychanalyse - Travaux 1967-1992. Paris: Flammarion. (Original publié en 1990).

Laplanche, J. (1997c). Masochisme et théorie de la séduction généralisée (pp. 439-456). In J. Laplanche. Le primat de l'autre en psychanalyse - Travaux 19671992. Paris: Flammarion. (Original publié en 1991).

Laplanche, J. (1999a). Court traité de l'inconscient (pp. 67-114). In J. Laplanche. Entre séduction et inspiration: l'homme. Paris: PUF. (Original publié en 1993).

Laplanche, J. (1999a). Les forces en jeu dans le conflit psychique (pp. 127-146). In J. Laplanche. Entre séduction et inspiration: l'homme. Paris: PUF. (Original publié en 1994).

Laplanche, J. (1999b). La psychanalyse comme antiherméneutique (pp. 243-262). In J. Laplanche. Entre séduction et inspiration: l'homme. Paris: PUF. (Original publié en 1994).

Laplanche, J. (2004). Les échecs de la traduction. Psychiatrie Française, 33(3-4): 69-91.
Laplanche, J. (2006). Problématiques VII: Le fourvoiement biologisant de la sexualité chez Freud. Paris: PUF. (Original publié en 1991).

Laplanche, J. (2007). Castration et Edipe comme codes et schémas narratifs (pp. 293-300). In J. Laplanche. Sexual: La sexualité élargie au sens freudien, 20002006. Paris: PUF.

M’Uzan, M. (1997). Affect et processus d'affectation (pp. 127-150). In M. M'Uzan. De l'art à la mort. Paris: Gallimard. (Original publié en 1970)

Marty, P., \& M’Uzan, M. (1963). La pensée opératoire. Revue Française de Psychanalyse. 27(n. Spécial): 345355.

McDougall, J. (1989). Théâtres du corps. Paris: Gallimard.

Press, J. (1995). La répression, refoulement du pauvre? Revue Française de Psychosomatique, 7: 123-142.

Rosé, D. (1997). L'endurance primaire. De la clinique psychanalytique de l'excitation à la théorie psychanalytique de l'excès. Paris: PUF.

Smadja, C. (2011). Le travail de psychisation du corps. Revue Française de Psychosomatique. 39:147-161.

Tarelho, L. C. (1999). Paranoïa et théorie de la séduction généralisée. Paris: PUF.

Recebido em 19-07-2012 Aceito em 15-12-2012
Endereço para correspondência:

Isabelle Gernet. Laboratoire de Psychologie Clinique et Psychopathologie (EA 4056), Institut de Psychologie, Université Paris Descartes - 71 Avenue Edouard Vaillant - F-92774 BOULOGNE BILLANCOURT Cedex - France.E-mail: igernet@free.fr. 